\title{
Role of Hcp, a type 6 secretion system effector, of Aeromonas hydrophila in modulating activation of host immune cells
}

\author{
Giovanni Suarez, Johanna C. Sierra, Michelle L. Kirtley \\ and Ashok K. Chopra \\ Department of Microbiology and Immunology, University of Texas Medical Branch, Galveston, \\ TX 77555, USA
}

Correspondence

Ashok K. Chopra

achopra@utmb.edu

Received 5 May 2010

Revised 11 August 2010

Accepted 23 August 2010

\begin{abstract}
Recently, we reported that the type 6 secretion system (T6SS) of Aeromonas hydrophila SSU plays an important role in bacterial virulence in a mouse model, and immunization of animals with the T6SS effector haemolysin co-regulated protein ( $\mathrm{Hcp}$ ) protected them against lethal infections with wild-type bacteria. Additionally, we showed that the mutant bacteria deleted for the vas $H$ gene within the T6SS gene cluster did not express the $h c p$ gene, while the vasK mutant could express and translocate $\mathrm{Hcp}$, but was unable to secrete it into the extracellular milieu. Both of these $A$. hydrophila SSU mutants were readily phagocytosed by murine macrophages, pointing to the possible role of the secreted form of Hcp in the evasion of the host innate immunity. By using the $\Delta$ vas $H$ mutant of $A$. hydrophila, our in vitro data showed that the addition of exogenous recombinant $\mathrm{Hcp}$ ( $\mathrm{rHcp}$ ) reduced bacterial uptake by macrophages. These results were substantiated by increased bacterial virulence when $\mathrm{rHcp}$ was added along with the $\Delta$ vas $H$ mutant in a septicaemic mouse model of infection. Analysis of the cytokine profiling in the intraperitoneal lavage as well as activation of host cells after $4 \mathrm{~h}$ of infection with the $\Delta$ vas $H$ mutant supplemented with rHcp indicated that this T6SS effector inhibited production of proinflammatory cytokines and induced immunosuppressive cytokines, such as interleukin-10 and transforming growth factor- $\beta$, which could circumvent macrophage activation and maturation. This mechanism of innate immune evasion by Hcp possibly inhibited the recruitment of cellular immune components, which allowed bacterial multiplication and dissemination in animals, thereby leading to their mortality.
\end{abstract}

\section{INTRODUCTION}

Aeromonas hydrophila is among the most common species associated with wound and soft tissue infections, gastroenteritis and septicaemia in the host (Chopra \& Houston, 1999; Janda \& Abbott, 1998). Our laboratory group and others have characterized several virulence factors from Aeromonas species, which are secreted via the type 2 and type 3 secretion systems (Carvalho-Castro et al., 2010; Chopra et al., 2000; Sha et al., 2005, 2007; Sierra et al.,

Abbreviations: 7-AAD, 7-amino actinomycin D; DC, dendritic cell; G-CSF, granulocyte colony-stimulating factor; IFN, interferon; IL, interleukin; i.p., intraperitoneal; KC, keratinocyte-derived chemokine; LPS, lipopolysaccharide; M-CSF, macrophage colony-stimulating factor; $\mathrm{MHC}$, major histocompatibility complex; MIP, macrophage inflammatory protein; MTT, 3-(4,5-dimethylthiazol-2-yl)-2,5-diphenyl tetrasodium bromide; SOCS, suppressor of cytokine signalling; T6SS, type 6 secretion system; TGF, transforming growth factor; Th, T helper [cell]; TNF, tumour necrosis factor; WT, wild-type.

A supplementary figure, showing the effect of $\mathrm{Hcp}$ and $\mathrm{rHcp}$ on bacterial growth and murine macrophages, is available with the online version of this paper.
2007; Tan et al., 2008; Vilches et al., 2009). Our most recent studies are focused on the type 6 secretion system (T6SS) and its effectors from diarrhoeal isolate SSU of $A$. hydrophila (Suarez et al., 2008, 2010). Although the T6SS is highly conserved in Proteobacteria, the general mechanism as to how this system operates remains poorly understood (Das \& Chaudhuri, 2003).

A. hydrophila SSU has one chromosomally located T6SS gene cluster which is regulated by the $\sigma^{54}$ activator encoded by the virulence-associated gene, vasH (Suarez et al., 2008). We reported that the $\Delta v a s H$ mutant of A. hydrophila SSU was unable to express genes encoding haemolysin-coregulated protein (Hcp) and the valine glycine repeat G (VgrG) family of proteins $\mathrm{VgrG} 2$ and $\mathrm{VgrG}$, which constitute part of the T6SS gene cluster (Suarez et al., 2008). However, this mutant was able to express, but was unable to secrete or translocate, VgrG1 that resides outside of the T6SS gene cluster (Suarez et al., 2010). Further, deletion of hcp or $\operatorname{vgrG}$ prevents secretion of the other, thereby demonstrating dual roles of Hcp and VgrG as structural components of the T6SS apparatus and as effector proteins (Cascales, 
2008). Importantly, both Hcp and VgrG proteins represent a hallmark of the T6SS secreted proteins in all of the bacteria that possess this system (Bingle et al., 2008; Cascales, 2008; Filloux et al., 2008; Pukatzki et al., 2009). Although the role of VgrG1 in bacterial virulence was convincingly demonstrated recently by us in A. hydrophila SSU (Suarez et al., 2010) and V. cholerae (Ma et al., 2009; Ma \& Mekalanos, 2010), the mechanism of Hcp in modulating the organism's virulence is poorly understood.

Innate immunity is the first line of host defence against the challenged organisms, and pattern recognition receptors sense different microbial ligands (known as pathogen-associated molecular patterns, PAMPs) (Barton \& Medzhitov, 2003; Janeway \& Medzhitov, 2002; Medzhitov \& Janeway, 1999; Taylor et al., 2005), resulting in the triggering of signalling cascades that determine the host immune response by modulating maturation, activation and recruitment of cellular effectors [e.g. neutrophils, macrophages, dendritic cells (DCs) and natural killer cells] (Henneke \& Golenbock, 2004; Hume, 2006; Medzhitov \& Janeway, 1999; Plüddemann et al., 2006).

Phagocytosis is crucial for both innate and adaptive immunity (Coombes et al., 2004; Henneke \& Golenbock, 2004; Medzhitov \& Janeway, 1999), and macrophages and DCs are professional antigen-presenting cells which act as tissue sentinels and are able to present antigens to naïve T-cells (Gordon \& Taylor, 2005; Hume, 2006, 2008). Bacteria have developed different mechanisms to avoid innate immunity ranging from their ability to avoid recognition by toll-like receptor-4 (Ernst et al., 1999; Hajjar et al., 2002; Kawasaki et al., 2004), altering antigenicity of surface molecules to avoid phagocytosis (Seifert, 1996), interfering with mitogen-activated protein kinase signalling cascades (Park et al., 2002; Sweet et al., 2007; Thiefes et al., 2006), modulating actin polymerization and apoptosis (Abrahams \& Hensel, 2006; Pujol \& Bliska, 2005; Ruckdeschel et al., 2002; Viboud \& Bliska, 2005), manipulating phagosome trafficking and maturation (Dramsi \& Cossart, 2002; Sturgill-Koszycki et al., 1994; Uchiya et al., 1999), and inducing the production of immunosuppressive cytokines, particularly interleukin (IL)10. The latter mechanism avoids activation of macrophages, maturation of DCs and recruitment of granulocytes (McGuirk et al., 2002; Sing et al., 2002; Zuany-Amorim et al., 2002).

Previously, we demonstrated that A. hydrophila SSU $\Delta v a s H$ and $\Delta$ vasK mutants were easily phagocytosed by murine RAW 264.7 macrophages compared with the phagocytosis of wild-type (WT) bacteria (Suarez et al., 2008). Further, we showed that the $\Delta v a s H$ mutant strain did not express the gene encoding Hcp and that the $\Delta$ vasK mutant was able to produce Hcp, but was unable to secrete it (Suarez et al., 2008). Hence, we hypothesized that the secreted form of Hcp could be playing a role in inhibition of phagocytosis of A. hydrophila SSU by macrophages. Here, we report that Hcp indeed played a role in modulating the innate immunity by inhibiting the phagocytosis of A. hydrophila
SSU, thus allowing its multiplication and spread to different organs of the host. Our results show that Hcp is able to bind to macrophages and induce the production of IL-10 and transforming growth factor (TGF)- $\beta$, affecting the activation and maturation of macrophages, and, consequently, the recruitment of other cellular immune components needed to clear bacterial infection.

\section{METHODS}

Cell line and bacterial strains. RAW 264.7, a murine macrophage cell line, was maintained in Dulbecco's modified eagle medium with high glucose (DMEM) (Invitrogen), supplemented with $10 \%$ fetal bovine serum (FBS) under standard cell culture growth conditions.

A. hydrophila SSU $\Delta$ act (act encodes the type 2 secretion system-secreted cytotoxic enterotoxin) (Xu et al., 1998) and $\Delta a c t / \Delta v a s H$ isogenic mutant strains were developed in the laboratory as described previously (Suarez et al., 2008). These mutants were grown in LB medium supplemented with kanamycin $\left(50 \mu \mathrm{g} \mathrm{ml}^{-1}\right)$ for $A$. hydrophila $\Delta a c t$, and kanamycin, streptomycin $\left(40 \mu \mathrm{g} \mathrm{ml}^{-1}\right)$ and spectinomycin $\left(50 \mu \mathrm{g} \mathrm{ml}^{-1}\right)$ for the $A$. hydrophila $\Delta$ act/DvasH mutant.

Recombinant Hcp protein. The $h c p$ ( $h c p 2$ residing within the T6SS cluster) gene was cloned in the pET-30a vector for the production of recombinant protein as reported previously (Suarez et al., 2008). rHcp containing a $6 \times$ His tag was purified by nickel affinity chromatography, dialysed against PBS, and then passed through a polymyxin column (Bio-Rad) to remove any residual lipopolysaccharide (LPS). The pass-through fraction was filtered by using a $0.2 \mu \mathrm{m}$ filter, and the protein concentration was quantified by using the Bradford assay (BioRad). The removal of LPS from rHcp was verified by the Limulus amebocyte lysate assay (Pyrosate-Dial Medical Supply), and the purity of rHcp was verified by Coomassie Blue staining of the gel.

Cell viability. To determine host cell viability (RAW 264.7 cells treated with $10 \mu \mathrm{g} \mathrm{rHcp} \mathrm{ml}{ }^{-1}$ for different time periods), we performed the 7amino actinomycin D (7-AAD) (Becton Dickinson) and the colorimetric 3-(4,5-dimethylthiazol-2-yl)-2,5-diphenyl tetrasodium bromide (MTT) (Chemicon) assays, as described previously (Sierra et al., 2007).

Conditioned medium. To obtain conditioned medium containing $\mathrm{Hcp}$, we grew the $\Delta$ act mutant strain of A. hydrophila SSU $\left(1 \times 10^{7}\right.$ c.f.u.) in $3 \mathrm{ml}$ DMEM supplemented with $0.5 \% \mathrm{FBS}$ at $37^{\circ} \mathrm{C}$. After $2 \mathrm{~h}$, the bacteria were removed by centrifugation, and the supernatant was passed through a $0.2 \mu \mathrm{m}$ membrane filter. The resulting medium was tested by Western blot analysis for the presence of Hcp by using specific antibodies, and used immediately in the phagocytosis assay.

Western blot analysis. Western blot analysis was performed to detect Hcp in the conditioned medium collected after infection of RAW 264.7 cells with various strains of $A$. hydrophila. Briefly, tissue culture supernatant was filtered by using a $0.2 \mu \mathrm{m}$ filter to remove bacteria; proteins in the supernatant were separated by using $15 \%$ SDS-PAGE. The proteins were then transferred to a nitrocellulose membrane which was blocked with $5 \%$ skimmed milk $+1 \%$ BSA overnight at $4{ }^{\circ} \mathrm{C}$. Mouse-anti-Hcp serum was used at a dilution of $1: 2000$ for $1 \mathrm{~h}$, and horseradish peroxidase-conjugated, goat-anti-mouse antibodies were used at a dilution of 1:5000 for $45 \mathrm{~min}$. The blot was then developed by using ECL-Western blotting detection reagent (GE Healthcare).

Phagocytosis assay. Briefly, $1.5 \times 10^{6}$ RAW 264.7 cells suspended in $1 \mathrm{ml}$ DMEM supplemented with $0.5 \%$ FBS were split into aliquots in polypropylene tubes $(12 \times 75 \mathrm{~mm})$. Different bacterial strains were added at an m.o.i. of 10 for $30 \mathrm{~min}$. Then, the cell suspension was 
centrifuged at $250 \mathrm{~g}$ for $5 \mathrm{~min}$ and the supernatant was removed. The pellet was resuspended in $500 \mu \mathrm{l}$ of DMEM plus $0.5 \%$ FBS (above) and $250 \mu \mathrm{g}$ gentamicin $\mathrm{ml}^{-1}$ (CellGro) to kill extracellular bacteria. After $2 \mathrm{~h}$, the macrophages were washed three times with $1 \mathrm{ml}$ DMEM to remove dead bacteria and gentamicin. The host cells were lysed in $500 \mu \mathrm{l}$ sterile water, and the cell lysates were plated on LB agar plates containing kanamycin $\left(50 \mu \mathrm{g} \mathrm{ml}{ }^{-1}\right)$ to determine c.f.u. (Suarez et al., 2008). We examined complete lysis of the host cells under the microscope before plating the cell lysates.

Animal survival. Groups of 10 Swiss Webster female mice (Charles River) were challenged via the intraperitoneal (i.p.) route with a sublethal dose of $A$. hydrophila $\Delta a c t / \Delta v a s H$ mutant, either alone or in combination with different amounts of $\mathrm{rHcp}(1-10 \mu \mathrm{g})$. As a control, a group of mice was challenged with either the highest dose of rHcp $(10 \mu \mathrm{g})$ or the $\Delta$ act parental strain. The survival of mice was followed for 16 days post-infection.

Bacterial spread. Mice were infected via the i.p. route with a sublethal dose of $A$. hydrophila $\Delta a c t / \Delta v a s H$ mutant alone or in combination with $\mathrm{rHcp}(10 \mu \mathrm{g})$. The animals were euthanized after $48 \mathrm{~h}$, and sections of liver, spleen and lungs were homogenized and used to determine bacterial burden (Agar et al., 2009).

Intraperitoneal lavage. After $4 \mathrm{~h}$ of challenge of mice via the i.p. route with a sublethal dose of $A$. hydrophila $\Delta a c t / \Delta v a s H$ mutant alone or in combination with $\mathrm{rHcp}$, the animals were euthanized, and the peritoneal cavity was flushed with $1.5 \mathrm{ml}$ sterile Hanks' solution (Invitrogen). The lavage was collected and centrifuged at $250 \mathrm{~g}$ for $5 \mathrm{~min}$. The supernatant was used for evaluating various cytokines/chemokines by the multiplex bead array, and the pellets containing host cells were used to determine their phenotypes by flow cytometry (see below).

Hcp-binding assay. The whole cell population from the intraperitoneal lavage of mice was incubated with $\mathrm{rHcp}\left(10 \mu \mathrm{g} \mathrm{ml}^{-1}\right)$ for $1 \mathrm{~h}$. Then, the cells were incubated for $30 \mathrm{~min}$ with anti-mouse CD16/ CD32 antibodies (Becton Dickinson). Next, the cells were dispensed into different tubes and incubated with pre-immune mouse serum $(1: 100)$ as an isotype control, or with the mouse anti-Hcp serum $(1: 100)$ for $1 \mathrm{~h}$. Subsequently, the host cells were incubated with Alexa Fluor 488-conjugated goat anti-mouse antibodies (Invitrogen) for $45 \mathrm{~min}$, followed by incubations with PE-Cy5-conjugated anti-mouse F4/80, PE-conjugated anti-mouse Gr-1 antibodies, and their respective isotype controls. Between steps, the cells were washed twice with chilled Hanks' solution, and all the incubations were performed on ice. The cells were acquired in a FACScanto (Becton Dickinson) flow cytometer and analysed by using FACSDiva software (Becton Dickinson).

Quantification of Hcp produced by the $\Delta$ act mutant of $\boldsymbol{A}$. hydrophila SSU. To determine the amount of Hcp produced by the parental A. hydrophila strain in the mouse peritoneum after $4 \mathrm{~h}$ of infection, we infected animals via the i.p. route with $3 \times 10^{7}$ bacterial cells per animal. Subsequently, the animals were euthanized and the intraperitoneal cavity was flushed with $3 \mathrm{ml}$ Hanks' solution. This lavage was filtered by using $0.2 \mu \mathrm{m}$ membrane filters and precipitated with $10 \%$ (final concentration) TCA. The protein pellet was resuspended in $500 \mu \mathrm{l}$ SDS loading buffer, and $20 \mu \mathrm{l}$ was separated by using $6-15 \%$ SDS-PAGE. In parallel, different concentrations of rHcp were loaded on a similar gel to build a standard curve. Proteins from both the gels were transferred to nitrocellulose membranes, and Western blot analysis was performed using anti-rHcp antibodies. Xray films obtained after exposure of the membranes were analysed by densitometry, and the concentration of Hcp present in the intraperitoneal lavage was determined from the standard curve.

Cytokines. The supernatants obtained after intraperitoneal lavages of mice (see Intraperitoneal lavage) were tested for cytokine/chemokine levels by a multiplex bead array (Millipore) following the manufacturer's instructions. The data were acquired and analysed in a Bioplex 200 system which uses Bioplex manager software v.5.0 (Bio-Rad).

We measured levels of TGF- $\beta$ in the intraperitoneal lavage supernatant samples by using an ELISA (eBiosciences) and following the manufacturer's instructions. The colour reaction was read in a microplate reader Versa-max (Molecular Devices).

Flow cytometry. Cell pellets collected after intraperitoneal lavages of mice (see Intraperitoneal lavage) were stained with a panel of antibodies conjugated with FITC (anti-CD11c, anti-CD69), PE (antiGr-1, anti-MHC-class II) and PE-Cy5 (anti-F4/80) for flow cytometry (eBiosciences). The cells were incubated with antibodies for $1 \mathrm{~h}$ and then washed twice with $1 \mathrm{ml}$ PBS and fixed with $2 \%$ paraformaldehyde. The cells were acquired in a FACScan and analysed by FACSdiva software (Becton Dickinson). Prior to incubation with antibodies, receptors Fc $\gamma$ III/II were blocked using anti-CD16/CD32 antibodies (BD Pharmingen) for $30 \mathrm{~min}$.

Statistical analysis. The data were analysed by one-way ANOVA and Tukey's post-test with GraphPad Prism version 4 software, and the animal survival curves were analysed by the Logrank test. At least three independent experiments were performed to represent biological replicates, unless otherwise stated.

\section{RESULTS}

\section{Hcp inhibits the phagocytosis of $A$. hydrophila SSU $\Delta$ act/AvasH mutant in vitro}

Since the secretion of Hcp was affected in both the $\Delta v a s H$ and $\Delta$ vasK mutant strains (Suarez et al., 2008), we hypothesized that the secreted form of Hcp could be playing a role in the bacterial inhibition of phagocytosis. To test this, we used conditioned medium from the $\Delta a c t$ parental strain of A. hydrophila which contained Hcp (Fig. 1a) in the phagocytosis assay. Consequently, RAW 264.7 murine macrophages were infected with the $\Delta a c t / \Delta v a s H$ mutant in the conditioned medium, thus allowing complementation of the mutant with exogenous Hcp protein. We noted that the ability of the A. hydrophila $\Delta a c t / \Delta v a s H$ mutant to be phagocytosed by macrophages was reduced in the presence of Hcp (Fig. 1b). To confirm that this effect was indeed due to Hcp and not to other secreted bacterial proteins, we performed a phagocytosis assay by using the $\Delta a c t / \Delta v a s H$ mutant in conjunction with different concentrations of purified $\mathrm{rHcp}$. We found that phagocytosis of the mutant by macrophages was inhibited by $\mathrm{rHcp}$ in a dose-dependent fashion, with statistically significant differences observed at rHcp doses of $5.6 \mu \mathrm{g}$ and greater (Fig. 1c) compared with mutant bacteria $(\Delta a c t / \Delta v a s H)$ alone without $\mathrm{rHcp}$.

To determine whether the doses of rHcp necessary to inhibit phagocytosis in our in vitro experiments were physiologically relevant, we measured the amounts of Hcp produced by the parental $\Delta a c t$ mutant strain of $A$. hydrophila in the mouse peritoneum and we found 8$10 \mu \mathrm{g} \mathrm{Hcp}$ after $4 \mathrm{~h}$ of infection, indicating physiological relevance of the rHcp doses used in this study (Fig. 1c). Our positive and negative controls in this assay included macrophages infected with the $\Delta a c t$ and $\Delta a c t / \Delta v a s H$ strains 

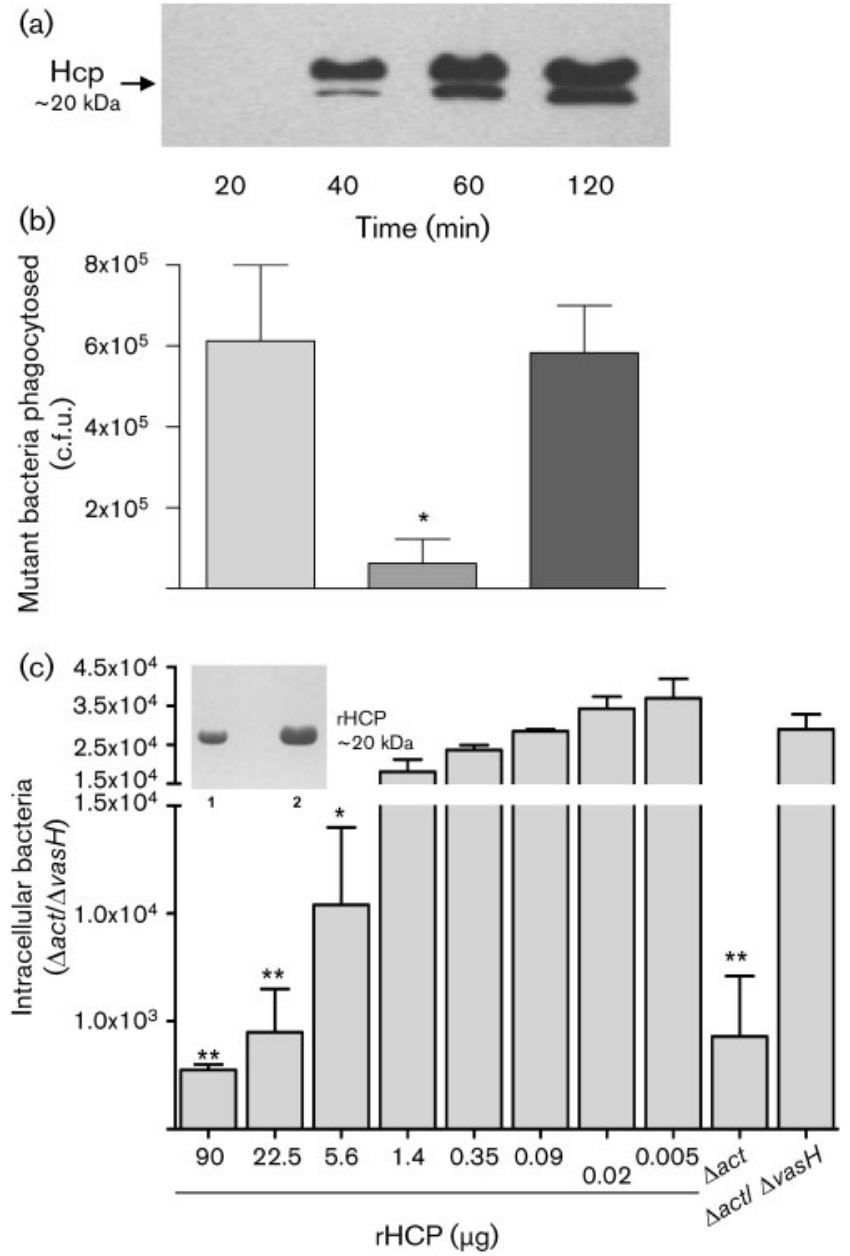

Fig. 1. Hcp inhibits phagocytosis of the $\Delta a c t / \Delta v a s H$ mutant of $A$. hydrophila SSU by RAW 264.7 murine macrophages. (a) Western blot analysis of the conditioned medium from the parental $\Delta$ act strain of $A$. hydrophila by using antibodies to Hcp. The presence of a doublet on the Western blot may represent the degradation product of Hcp. (b) Conditioned medium containing Hcp inhibits phagocytosis of the $\Delta a c t / \Delta v a s H$ mutant. Phagocytosis assay using the $A$. hydrophila $\Delta a c t / \Delta v a s H$ mutant and RAW 264.7 cells was performed in conditioned medium from $A$. hydrophila SSU $\Delta$ act parental strain as the exogenous source of $\mathrm{Hcp}$ (mid-grey). We also used conditioned medium from $A$. hydrophila $\Delta$ act/ $\Delta$ vas $H$ mutant as a control for any other secreted proteins/factors independent of the T6SS that could affect phagocytosis (dark grey). Another control included fresh DMEM supplemented with $0.5 \%$ FBS (light grey). Data shown are the mean \pm SD of three independent experiments. (c) $\mathrm{rHcp}$ inhibits phagocytosis of the $A$. hydrophila $\Delta \mathrm{act} / \Delta \mathrm{vas} H$ mutant. As a control, we included $A$. hydrophila $\Delta$ act/ $\Delta$ vas $H$ mutant without $\mathrm{rHcp}$, as well as the $A$. hydrophila $\Delta$ act parental strain. Data shown are the mean $\pm S D$ of three independent experiments. The statistical difference was calculated by a one-way ANOVA test. ${ }^{\star} P<0.05 ;{ }^{* \star} P<0.01$. The Coomassie Blue stained gel (inset) shows the purity of $\mathrm{rHcp}$ obtained after purification and removal of LPS. The lanes were loaded with $5 \mu \mathrm{g}$ (lane 1) and $10 \mu \mathrm{g}$ (lane 2) of $\mathrm{rHcp}$. The physiological amount of Hcp produced by the parental strain of $A$. hydrophila was up to $8-10 \mu \mathrm{g}$ in the peritoneum after infection. of A. hydrophila, respectively. As expected, the former showed minimal phagocytosis, while the latter exhibited much higher levels of phagocytosis by macrophages. These data clearly indicate that the secreted Hcp plays a role in bacterial phagocytosis. We confirmed that the difference in phagocytosis between the parental $\Delta a c t$ strain of $A$. hydrophila and that of the $\Delta a c t / \Delta v a s H$ mutant was not due to differences in their growth rates (Supplementary Fig. S1a, available with the online version of this paper) or to toxic effects of rHcp on RAW 264.7 cells for at least up to $6 \mathrm{~h}$, as determined by MTT and 7-AAD assays (Supplementary Fig. S1b, c), and as well as by microscopic visualization of host cells (data not shown). The purity of rHcp was evaluated by Coomassie-Blue-staining of the gel (Fig. 1c, inset). In addition, we tested $\mathrm{rHcp}$ for any residual LPS and found less than 1 endotoxin unit in $10 \mu \mathrm{g}$ of the recombinant protein, which has no influence on macrophage activation.

\section{Hcp decreases the survival rate of mice infected with the $\Delta a c t / \Delta$ vasH mutant of $A$. hydrophila SSU}

Since the secreted form of Hcp decreased bacterial phagocytosis, and we showed earlier that mice infected via the i.p. route with the $\Delta v a s H$ mutant had a better survival rate compared with mice infected with the WT strain (Suarez et al., 2008), we hypothesized that bacteria producing Hcp would have a better chance of evading the innate immunity and causing systemic effects. Hence, we challenged mice with a sublethal dose of the $\Delta a c t / \Delta v a s H$ mutant together with $\mathrm{rHcp}$ at different concentrations and monitored deaths for 16 days. We found that the addition of rHcp decreased the survival rates of mice after infection with the $\Delta a c t / \Delta$ vasH mutant, with $100 \%$ of the mice dying with 5 and $10 \mu \mathrm{g}$ of rHcp (Fig. 2). These data indicated complementation of the above mutant with exogenous rHcp in terms of bacterial virulence.

\section{Hcp enhances the spread of the $\Delta a c t / \Delta v a s H$ mutant of $\boldsymbol{A}$. hydrophila SSU in a mouse model}

Since our data showed that the presence of rHcp increased the death rate of mice infected with sublethal doses of the $\Delta a c t / \Delta v a s H$ mutant, we evaluated the bacterial load in mouse organs after $48 \mathrm{~h}$ of infection in the presence of rHcp. Our results indicated that rHcp allowed the mutant bacteria to spread more efficiently to different organs (lungs, livers and spleens) possibly resulting in the animals' death due to a systemic effect (Fig. 3). These bacterial numbers in different organs of mice infected with the $\Delta a c t /$ $\Delta v a s H$ mutant in the presence of $\mathrm{rHcp}$ were very similar to their numbers reported recently from mouse organs after infection with the $\Delta a c t$ parental strain of A. hydrophila (Sierra et al., 2010). Our results supported the prediction that Hcp played a role in innate immunity by avoiding bacterial clearance in the peritoneal cavity by phagocytosis, thus allowing organisms to multiply and spread to various organs. 


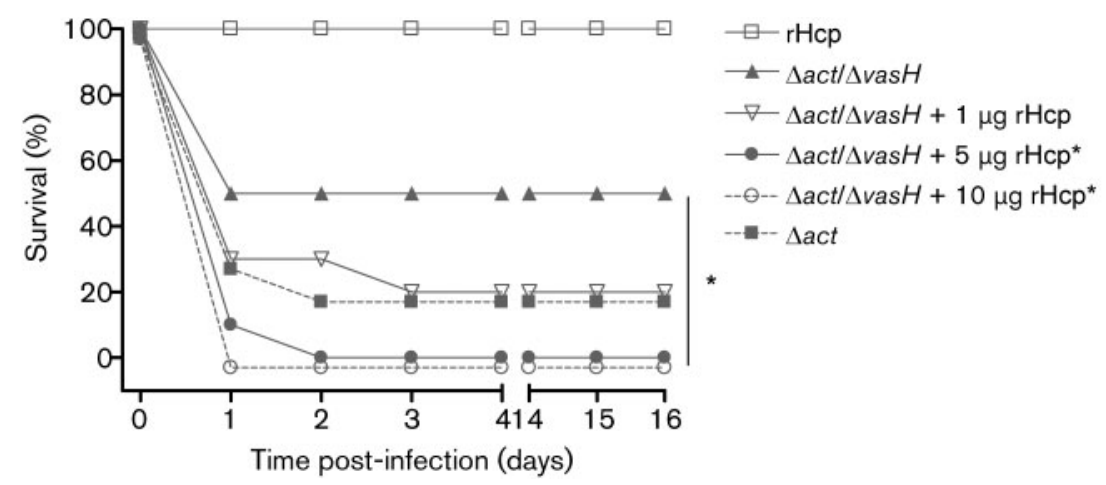

Fig. 2. $\mathrm{rHcp}$ increases the virulence of the $A$. hydrophila SSU $\Delta a c t / \Delta v a s H$ mutant. Survival curves of mice $(n=10)$ infected via the i.p. route with $8 \times 10^{6}$ c.f.u. of $A$. hydrophila $\Delta$ act/ $\Delta$ vasH mutant in the presence of different concentrations of $\mathrm{rHcp}$. As a control, groups of mice $(n=10)$ were infected with the same doses of the mutant bacteria without $\mathrm{rHcp}$ or the $\Delta$ act parental strain. As another control for rHcp toxicity, mice were inoculated with the highest concentration of $\mathrm{rHcp}$ without the bacteria. The deaths were recorded for 16 days after infection. Asterisks represent groups of mice with statistically significant differences in mortality against the same doses of bacteria without the $\mathrm{rHcp}, P<0.05$. Each animal experiment was performed in duplicate and a representative experiment is shown.

\section{Hcp binds to intraperitoneal immune cells of mice}

Our earlier study showed that Hcp present in the conditioned medium of the WT strain of A. hydrophila SSU was able to bind RAW 264.7 macrophages (Suarez et al., 2008). To confirm that Hcp also binds to primary macrophages, we collected whole cell populations from the peritoneal cavity of naïve mice and, after incubating them with rHcp ex vivo, we performed a multicoloured flow cytometry analysis by using antibodies against Hcp, F4/80 and Gr-1. Fig. 4(a) shows the forward scatter versus the side scatter plot from the whole cell population isolated after the lavage. For analysis, we evaluated rHcp binding to cells gated on F4/80 (macrophages) (Fig. 4b) and on Gr-1 (granulocytes) (Fig. 4c). As can be seen, rHcp was able to

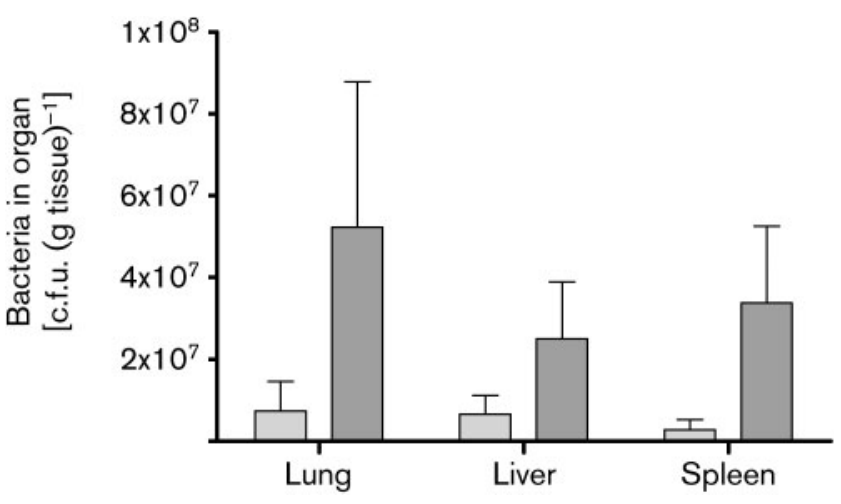

Fig. 3. $\mathrm{rHcp}$ plays a role in the spread of $A$. hydrophila SSU $\Delta$ act/ $\Delta$ vas $H$ mutant to different mouse organs. Bacterial burden was measured in the lungs, livers and spleens $48 \mathrm{~h}$ after i.p. infection with $3 \times 10^{7}$ c.f.u. $A$. hydrophila $\Delta$ act/ $\Delta$ vas $H$ mutant in the presence of $\mathrm{rHcp}(10 \mu \mathrm{g})$ (mid-grey). Mice infected with the same dose of bacteria without $\mathrm{rHcp}$ were used as a control (light grey). The data were normalized with the organ weights. The graph represents data from 10 mice per group. Error bars show SD. bind macrophages (Fig. 4d) and granulocytes (Fig. 4e), although the differential shift of the curve for granulocytes indicated that $\mathrm{rHcp}$ binding to this particular cell type was much less compared with its isotype control and that for the macrophages.

\section{Hcp modulates the expression of activation markers on intraperitoneal immune cells}

Since our data indicated that $\mathrm{rHcp}$ binds to cells involved in the innate immune response, we analysed, by flow cytometry, the status of these cells after $4 \mathrm{~h}$ of infection with the $\Delta a c t / \Delta v a s H$ mutant of $A$. hydrophila given along with two different doses of rHcp. Specifically, we analysed changes in the percentage of macrophages (F4/80), granulocytes (Gr-1) and DCs (CD11c). In addition, we examined the expression of CD69 (an early activation marker) and the major histocompatibility complex (MHC) class II. As controls, we used lavages from uninfected (given only PBS) mice, infected with the $\Delta a c t / \Delta v a s H$ mutant of $A$. hydrophila alone along with PBS, and injected via the i.p. route with $\mathrm{rHcp}$ alone.

We noted a significant increase in the percentage of Gr-1positive cells in the peritoneal cavity of animals infected with the $\Delta a c t / \Delta v a s H$ mutant when compared with the uninfected mice (Fig. 5a). However, this difference was independent of the presence of rHcp. Additionally, our results showed no significant changes in the percentage of macrophages and DCs in the peritoneal cavity after $4 \mathrm{~h}$ of infection, although we noted marginal increases in CD11cpositive cells (not statistically significant) related to the increasing concentrations of rHcp (Fig. 5a).

We then analysed the expression of CD69 and MHC class II in the total cell population as well as in cells gated for F4/ 80, Gr-1 and CD11c after cells were infected with the $\Delta a c t /$ $\Delta$ vasH mutant and given rHcp. As shown in Fig. 5(b), the percentage of CD69 in F4/80- and Gr-1-positive cells 
(a)

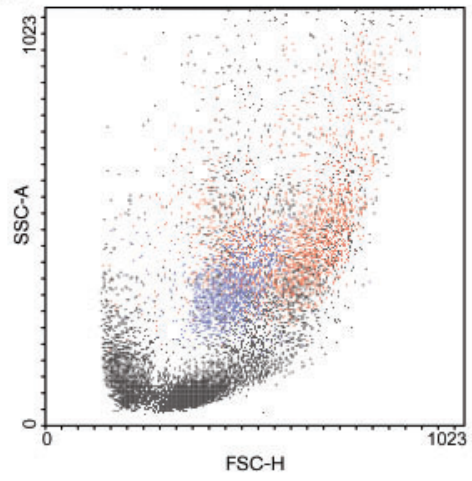

(b)

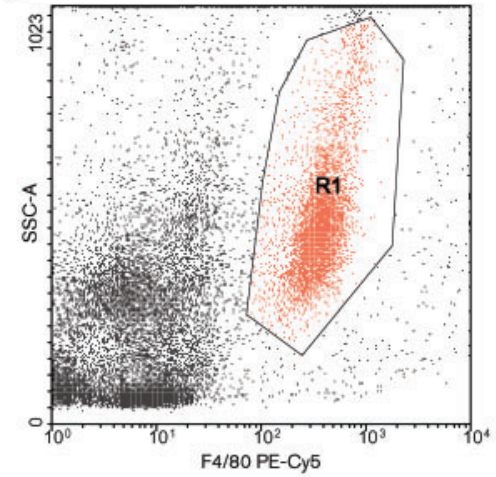

(c)

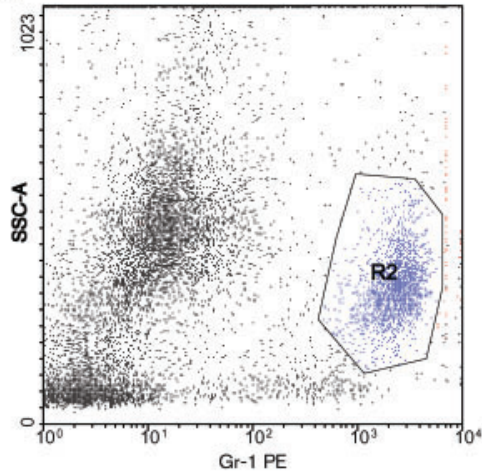

(d)

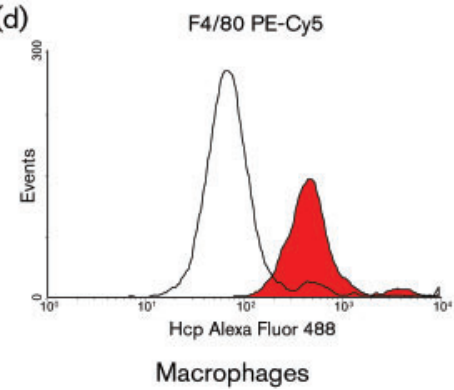

(e)

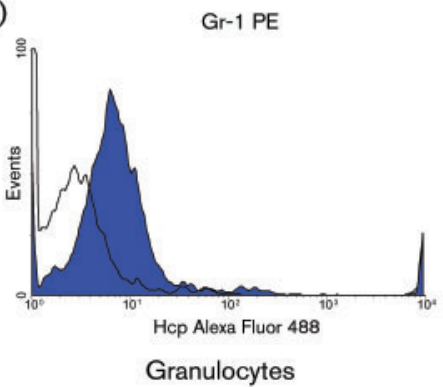

Fig. 4. $\mathrm{rHcp}$ binds to intraperitoneal immune cells. Total intraperitoneal cells were recovered after peritoneal lavages and incubated with $\mathrm{rHcp}(10 \mu \mathrm{g})$ ex vivo. Subsequently, the cells were stained by using antibodies against Hcp (FITC), Gr-1 (PE) and F4/80 (PE-Cy5). (a) Side scatter versus forward scatter plot for the total intraperitoneal cells. (b) Side scatter versus F4/80 $\mathrm{PE}-\mathrm{Cy} 5$ plot from the total cell population gated for the F4/80 positive cells (red). (c) Side scatter versus Gr-1 PE plot from the total cell population gated for the Gr-1-positive cells (blue). (d) A histogram plot showing staining for Hcp (Alexa Fluor 488) in cells which were F4/80 positive. The empty curve represents the isotype control, while the filled curve (red) represents cells incubated with anti-Hcp antibodies. (e) A histogram plot showing staining for Hcp (Alexa Fluor 488) in cells that were Gr-1 positive. The empty curve represents the isotype control and the filled curve (blue) represents cells incubated with anti-Hcp antibodies.

decreased, and these decreases were dependent on the dose of rHcp used. Likewise, the percentage of MHC class IIpositive cells in $\mathrm{F} 4 / 80$ cells decreased after mice were infected with the $\Delta a c t / \Delta v a s H$ mutant and given the higher dose of rHcp. However, the expression of MHC-class II showed an increase in CD11c cells (with $10 \mu \mathrm{g} \mathrm{rHcp}$ ), although the data were not statistically significant (Fig. 5c). Likewise, injection of rHcp alone did not significantly change the percentage of CD69 and MHC class II in any of the cell types analysed compared with the uninfected group of mice.

\section{Hcp modifies cytokine/chemokine production profiles induced by the A. hydrophila SSU $\Delta$ act/ $\Delta$ vasH mutant}

Since $\mathrm{rHcp}$ modulates the expression of activation markers on macrophages, granulocytes and DCs, we analysed cytokine/chemokine patterns induced by the $\Delta a c t / \Delta v a s H$ mutant in the peritoneal cavity after $4 \mathrm{~h}$ of infection (Table 1 and Fig. 6). We found that infection of mice with the mutant induced the production of a wide range of pro- and antiinflammatory cytokines/chemokines and growth factors.
However, the addition of rHcp, along with the $\Delta a c t / \Delta v a s H$ mutant, during infection significantly modified the levels of some of these cytokines/chemokines. Thus, the concentrations of IL-2, IL-10, IL-15, IL-12p70, macrophage inflammatory protein (MIP)-1 $\beta$, MIP-2, granulocyte colonystimulating factor (G-CSF), IL-6, and keratinocyte-derived chemokine $(\mathrm{KC})$ were increased by adding $\mathrm{rHcp}$; however, the production of gamma interferon (IFN- $\gamma$ ), IL- $1 \alpha$ and macrophage colony-stimulating factor (M-CSF) was inhibited (Table 1 and Fig. 6). On the other hand, intraperitoneal lavages from mice injected with $\mathrm{rHcp}$ alone showed low or non-detectable levels of cytokines/chemokines (Table 1).

\section{Hcp induces an alternative pathway of macrophage activation}

Activation of macrophages is an important mechanism in innate immunity against foreign invading organisms. There are two types of macrophage activation. M1 or classical activation is mainly a pro-inflammatory or $\mathrm{T}$ helper $(\mathrm{Th}) 1$ like response focused on removal and clearing of the infection or debris produced by an injury, and M2 or 

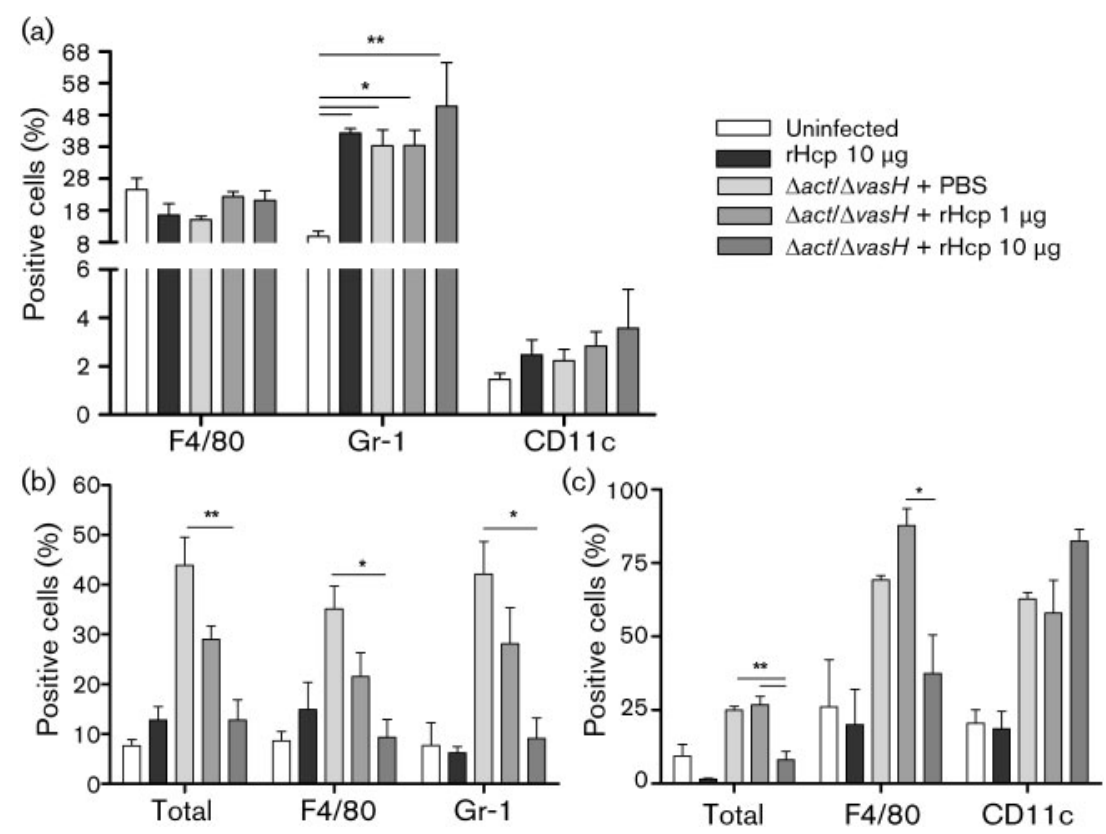

\begin{abstract}
Fig. 5. $\mathrm{rHcp}$ modulates the levels of activation markers on intraperitoneal innate immune cells of mice. Flow cytometric analysis of intraperitoneal cells recovered from lavages $4 \mathrm{~h}$ after infection with $3 \times 10^{7}$ c.f.u. A. hydrophila SSU $\Delta$ act/ $\Delta$ vas $H$ mutant in the presence of different doses of rHcp (1 and $10 \mu \mathrm{g}$ ). The PBS group represents animals challenged with only bacteria and PBS in place of $\mathrm{rHcp}$. (a) Percentage of positive cells for F4/80 (macrophages), Gr1 (granulocytes) and CD11c (DCs). (b) and (c) Percentage of positive cells expressing CD69 (b) and MHC class II (c) for the total population or for cells gated for F4/80, Gr-1 or CD11c. Statistically significant differences were measured by the one-way ANOVA test followed by Tukey's test. ${ }^{\star} P<0.05 ;{ }^{\star \star} P<0.01$. Error bars indicate SD.
\end{abstract}

alternative activation is focused more on tissue remodelling and wound healing. The latter is characterized by a low infiltration of cellular components and a Th2-like cytokine phenotype. Our data indicated that the presence of $\mathrm{rHcp}$ during infection with the $\Delta a c t / \Delta v a s H$ mutant could modulate an alternative pathway of macrophage activation, which is supported by decreases in some pro-inflammatory cytokine levels, such as IFN- $\gamma$ and IL- $1 \alpha$, and increases in anti-inflammatory cytokines, such as TGF- $\beta$, IL-6, IL-9 and IL-10 (Table 1 and Fig. 6). These results were also supported by the low levels of M-CSF and tumour necrosis factor (TNF) $\alpha$ induced by infection with the $\Delta a c t / \Delta v a s H$ mutant in mice, which remained unaltered in the presence of rHcp (Table 1 and Fig. 6). Taken together, these results correlated with the minimal macrophage recruitment in the peritoneal cavity of mice after infection with the $\Delta a c t /$ $\Delta$ vasH mutant of $A$. hydrophila SSU (Fig. 5a, F4/80 panel).

\section{DISCUSSION}

In this report, we described the role of the secreted form of Hcp in modulating the innate host immune response, specifically by inhibiting phagocytosis by macrophages. It has been reported that some bacterial proteins are able to interfere with phagocytosis by inducing host cell apoptosis, especially via caspase-1 activation (Abrahams \& Hensel, 2006; Diacovich \& Gorvel, 2010). We also showed that episomal expression of the $h c p$ gene in epithelial cells induced apoptosis (Suarez et al., 2008); however, there are no available data to show whether extracellular Hcp has any effect on the viability of eukaryotic cells. Based on 7AAD and MTT assays, we did not detect any significant toxicity in macrophages which could be associated with rHcp until up to $24 \mathrm{~h}$ of incubation, except for a small increase in mitochondrial activity (measured by MTT) detected after $2 \mathrm{~h}$ of incubation. We believe this initial increase was due to the activation of macrophages by $\mathrm{rHcp}$. Also, we found an increase in the percentage of 7-AADpositive cells ( $\sim 6 \%$ of the total population) as well as a decrease in the mitochondrial activity at $24 \mathrm{~h}$ that could be related to cell death after stimulation with $\mathrm{rHcp}$. Since our experiments did not require incubation times longer than $4 \mathrm{~h}$, we consider that the inhibition of phagocytosis in vitro due to Hcp present in the medium was independent of any toxic effects of Hcp on macrophages. Additionally, growth curves of the A. hydrophila $\Delta a c t$ and $\Delta a c t / \Delta v a s H$ mutant strains were similar, indicating that differences in phagocytosis of the A. hydrophila $\Delta a c t / \Delta v a s H$ mutant compared with that of its parental strain were not related to any differences in growth rates caused by the deletion of the $v a s H$ gene.

There are several examples of bacterial proteins that target phagocytosis at different levels in order to establish an infection. Our results showed that the secretion of Hcp into the extracellular medium played an important role in inhibiting innate immunity mediated by macrophages in vitro. Similarly, the presence of $\mathrm{rHcp}$ during infection increased bacterial virulence and allowed bacterial spread to different mouse organs after infection with the $A$. hydrophila $\Delta a c t / \Delta v a s H$ mutant. These data indicated that the increased virulence of this mutant in the presence of Hcp was associated with the inhibition of phagocytosis.

Macrophages, immature DCs and granulocytes are the main components of innate immunity and the first line of defence against invading organisms. Previously, we reported that the secreted form of Hcp binds RAW 264.7 macrophages (Suarez et al., 2008). In this report, we extended this observation and tested the binding of $\mathrm{rHcp}$ to primary intraperitoneal cells. We found that $\mathrm{rHcp}$ bound mainly to macrophages, although some binding to granulocytes was 
Table 1. Cytokine/chemokine levels in the peritoneal lavages of mice

Concentrations are in $\mathrm{pg} \mathrm{ml}^{-1}$; mean $\pm \mathrm{SD}$. ND, Not detected.

\begin{tabular}{|c|c|c|c|}
\hline \multirow[t]{2}{*}{ Cytokine/chemokine ${ }^{\star}$} & \multicolumn{2}{|c|}{ A. hydrophila SSU $\Delta a c t / \Delta v a s H$ mutant } & \multirow[t]{2}{*}{ rHcp $(10 \mu \mathrm{g})$} \\
\hline & + PBS & $+\operatorname{rHcp}(10 \mu \mathrm{g})$ & \\
\hline Eotaxin & $1018 \pm 58$ & $1088 \pm 35$ & $120 \pm 38$ \\
\hline G-CSF & $126849 \pm 18670$ & $184895 \pm 11335 \dagger$ & $434 \pm 108$ \\
\hline GM-CSF & $128 \pm 12$ & $142 \pm 24$ & $13 \pm 2$ \\
\hline IFN- $\gamma$ & $414 \pm 70$ & $183 \pm 39 \$$ & $\mathrm{ND}$ \\
\hline IL- $1 \alpha$ & $276 \pm 11$ & $240 \pm 14 \dagger$ & $18 \pm 5$ \\
\hline IL- $1 \beta$ & $811 \pm 68$ & $872 \pm 90$ & ND \\
\hline IL-2 & $12 \pm 3$ & $27 \pm 12 \ddagger$ & $\mathrm{ND}$ \\
\hline IL-3 & $\mathrm{ND}$ & ND & $\mathrm{ND}$ \\
\hline IL-4 & $\mathrm{ND}$ & $\mathrm{ND}$ & $\mathrm{ND}$ \\
\hline IL-5 & $129 \pm 22$ & $100 \pm 18$ & $\mathrm{ND}$ \\
\hline IL-6 & $62608 \pm 11044$ & $118731 \pm 22410 \dagger$ & $398 \pm 105$ \\
\hline IL-7 & $\mathrm{ND}$ & $\mathrm{ND}$ & $\mathrm{ND}$ \\
\hline IL-9 & $141 \pm 11$ & $209 \pm 6 \ddagger$ & $49 \pm 5$ \\
\hline IL-10 & $109 \pm 32$ & $192 \pm 32 \dagger$ & $\mathrm{ND}$ \\
\hline IL-12p40 & ND & $\mathrm{ND}$ & $\mathrm{ND}$ \\
\hline IL-12p70 & $123 \pm 3$ & $133 \pm 3 \dagger$ & $8 \pm 2$ \\
\hline IL-13 & $\mathrm{ND}$ & $\mathrm{ND}$ & $\mathrm{ND}$ \\
\hline IL-15 & $14 \pm 1$ & $20 \pm 0.5 \S$ & $\mathrm{ND}$ \\
\hline IL-17 & $1332 \pm 112$ & $1774 \pm 259$ & $\mathrm{ND}$ \\
\hline IP-10 & $413 \pm 33$ & $395 \pm 19$ & $198 \pm 73$ \\
\hline $\mathrm{KC}$ & $19104 \pm 4320$ & $36500 \pm 6353 \dagger$ & $47 \pm 27$ \\
\hline LIF & $\mathrm{ND}$ & $\mathrm{ND}$ & $\mathrm{ND}$ \\
\hline LIX & $560 \pm 55$ & $568 \pm 11$ & $38 \pm 10$ \\
\hline MCP-1 & $16108 \pm 1562$ & $17860 \pm 711$ & $200 \pm 73$ \\
\hline M-CSF & $12 \pm 2$ & $7 \pm 2 \ddagger$ & $\mathrm{ND}$ \\
\hline MIG & $2180 \pm 173$ & $1919 \pm 96$ & $55 \pm 23$ \\
\hline MIP- $1 \alpha$ & $401 \pm 29$ & $481 \pm 18$ & $86 \pm 7$ \\
\hline MIP-1 $\beta$ & $526 \pm 76$ & $734 \pm 61 \dagger$ & $60 \pm 17$ \\
\hline MIP-2 & $6288 \pm 935$ & $10754 \pm 633 \dagger$ & $20 \pm 5$ \\
\hline RANTES & $207 \pm 15$ & $238 \pm 17$ & $14 \pm 3$ \\
\hline TNF- $\alpha$ & $16 \pm 2$ & $22 \pm 3$ & ND \\
\hline VEGF & $\mathrm{ND}$ & $\mathrm{ND}$ & $\mathrm{ND}$ \\
\hline
\end{tabular}

${ }^{*}$ GM-CSF, granulocyte-macrophage colony-stimulating factor; LIF, leukaemia inhibitory factor; LIX, LPXinducible CXC chemokine; MCP, monocyte-chemoattractant protein; MIG, monocyte induced by IFN- $\gamma$; VEGF, vascular endothelial growth factor.

$\dagger P<0.05$ compared with + PBS.

$\ddagger P<0.01$ compared with + PBS.

$\S P<0.001$ compared with + PBS.

also detected. We speculate that differences in Hcp binding between macrophages and granulocytes could be related to differences in the Hcp-binding receptor(s) on these two cell types. Additionally, we tested binding of $\mathrm{rHcp}$ to human epithelial cell lines, such as HT-29 and HeLa. We found that rHcp bound to HeLa and HT-29 cells at levels that were five and nine times lower, respectively, compared with RAW cells (data not shown). Together, these data suggest that macrophages are the major cell target for Hcp.

Analysis of cytokine/chemokine levels in peritoneal lavages showed increases, dependent on the dose of $\mathrm{rHcp}$ used, in those involved in granulocyte/neutrophil recruitment and maturation, such as G-CSF, KC and MIP-2. These data may suggest that Hcp does not affect the recruitment of granulocytes in the peritoneal cavity of mice after infection with the $\Delta a c t / \Delta v a s H$ mutant; however, reduction in CD69 levels on the surface of granulocytes in an rHcp dosedependent manner indicates that this T6SS effector could have an effect on granulocyte activation.

The percentage of DCs (CD11c) was somewhat increased in mice infected with the $\Delta a c t / \Delta v a s H$ mutant in the presence of $\mathrm{rHcp}$. Importantly, these cells also showed 

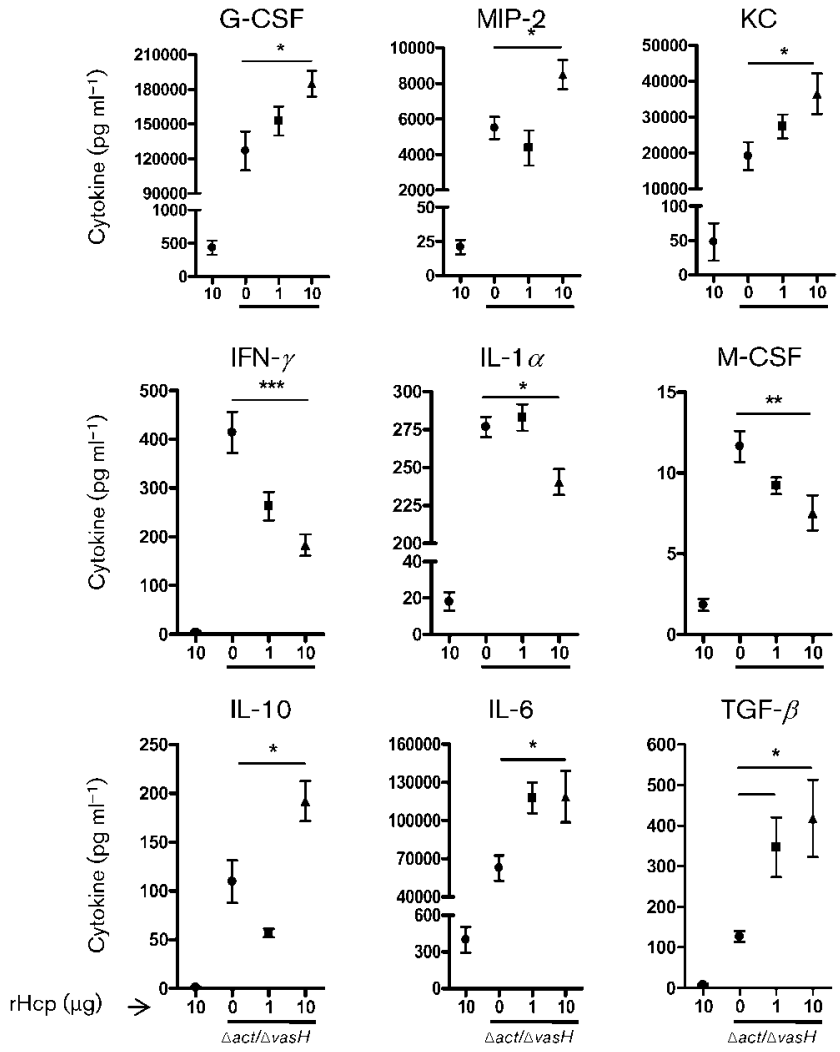

Fig. 6. $\mathrm{rHcp}$ induces an alternative pathway of macrophage activation in the peritoneal lavages of mice infected with $3 \times 10^{7}$ c.f.u. A. hydrophila SSU $\Delta a c t / \Delta v a s H$ mutant in the presence of different doses of rHcp ( 1 and $10 \mu \mathrm{g}$ ), as determined by cytokine/ chemokine profiling. Cytokines were measured by multiplex bead array on samples of intraperitoneal cavity lavages $4 \mathrm{~h}$ after infection. Statistically significant differences were measured by the one-way ANOVA test, followed by Tukey's test. ${ }^{\star} P<0.05$; ${ }^{* \star} P<0.01 ;{ }^{* \star *} P<0.001$. Error bars indicate SD.

increases (although not reaching statistical significance) in the percentage of the MHC class II molecules in the presence of rHcp. These results led us to suggest that Hcp might be inducing the maturation of DCs $\left(\mathrm{CD} 11 \mathrm{c}^{+} / \mathrm{MHC}\right.$ class $\mathrm{II}^{+}$); nonetheless, low levels of IL-12p70 and IFN- $\gamma$, and induction of IL-10 by rHcp in mice infected with the $\Delta a c t / \Delta v a s H$ mutant would hamper their normal activation and induction of regulatory phenotypes.

Macrophage activation is an important step in the modulation of immune responses, and a subdivision of alternative activation (M2) called 'deactivation' is mediated by the production of immunosuppressive cytokines IL-10 and TGF- $\beta$, downregulation of MHC class II molecules and pro-inflammatory cytokines/chemokines, low levels of IL-4 and IL-13 production, and finally low levels of production of reactive oxygen and nitrogen intermediates (Gordon, 2003; Hornef et al., 2002; Moore et al., 1993). This kind of activation could predispose the host to infection by the induction of regulatory cells which are inhibitors of inflammation, even in the presence of inflammatory cytokines/chemokines (Gordon \& Taylor, 2005; Mantovani et al., 2004; Mosser, 2003; Mosser \& Edwards, 2008). In accordance, we found that the pattern of cytokines/ chemokines in the peritoneal cavity after $4 \mathrm{~h}$ of infection with the A. hydrophila $\Delta a c t / \Delta v a s H$ mutant in the presence of $\mathrm{rHcp}$, was indicative of an alternative pathway of activation or 'deactivation' of macrophages led by the production of IL-10 and TGF- $\beta$, decreases in IFN- $\gamma$, low levels of TNF- $\alpha$, and no detection of IL-4 and IL-13.

IL-6 is an important modulator of the immune response due to its dual role as a Th1 cytokine, inducing the recruitment of cellular components, or as a Th2 cytokine, inhibiting the production of IFN- $\gamma$ and enhancing the production of IL-10 by induction of suppressor of cytokine signalling (SOCS) proteins (Diehl \& Rincon, 2002; Dong et al., 2009). In our study, we found that the $\Delta a c t / \Delta v a s H$ mutant induced the secretion of high levels of IL- 6 in the intraperitoneal cavity, which was even higher when $\mathrm{rHcp}$ was present. We believe that IL-6 together with IL-10 and TGF- $\beta$, and low levels of IFN- $\gamma$ and TNF- $\alpha$, has a role in the differentiation of immune cells present in the peritoneal cavity by induction of SOCS- 1 and -3 . The SOCS family of regulators are involved in the suppression of nuclear factor- $\kappa \mathrm{B}$ signalling pathways, as well as in promoting IL10 production (Dong et al., 2009). On the other hand, TGF- $\beta$, IL-9 and IL-10 also promote the production of SOCS proteins, which have been associated with impaired production of TNF- $\alpha$, downregulation of nitric oxide synthase, and the expression of the IL-1ra antagonist gene (Berlato et al., 2002; Lejeune et al., 2001).

We previously reported that immunization of mice with $\mathrm{rHcp}$ conferred protection against future infections with lethal doses of WT A. hydrophila SSU (Suarez et al., 2008). Overall, the results reported in this study highlighted the importance of $\mathrm{Hcp}$ in early stages of A. hydrophila infection. Therefore, adaptive immunity mediated by antibodies could neutralize the effect that Hcp has on inhibition of phagocytosis and, thus, enhance bacterial clearing by opsonization-mediated phagocytosis.

We also have characterized other A. hydrophila SSU toxins with enzymic activities that could impair phagocytosis. For example, VgrG1, a T6SS effector protein, has actin-ADPribosylation activity (Suarez et al., 2010), and the type 3 secretion system effector protein AexU, has ADP-ribosyltransferase and Rho-GAP activities (Sha et al., 2007; Sierra et al., 2007, 2010). Overall, A. hydrophila SSU has developed multiple mechanisms to circumvent innate immunity in order to establish an infection, and the role for each of these mechanisms in the disease process may depend on the host environment as well as the stage of infection.

In summary, Hcp binds to macrophages and induces the production of immunosuppressive cytokines IL-10 and TGF- $\beta$ which results in impaired recruitment and inhibition of phagocytosis. This is the first report, to our 
knowledge, that highlights how T6SS effector Hcp modulates the activation of macrophages to cause systemic infection in a mouse model. We focused our study on only one isolate of $A$. hydrophila. Whether other species of Aeromonas harbouring the T6SS behave similarly needs to be further elucidated.

\section{ACKNOWLEDGEMENTS}

This work was supported by the grants from NIH/NIAID (AI041611) and the Environmental Protection Agency. J.C.S. and G.S. were supported by J.W. McLaughlin Endowment Pre-doctoral Fellowships. J.C.S. was also supported by the Vale-Asche fellowship, University of Texas Medical Branch at Galveston. We thank Mark Griffin for his proficiency in flow cytometry acquisition data. We thank Ms Mardelle Susman for editorial assistance.

\section{REFERENCES}

Abrahams, G. L. \& Hensel, M. (2006). Manipulating cellular transport and immune responses: dynamic interactions between intracellular Salmonella enterica and its host cells. Cell Microbiol 8, 728-737.

Agar, S. L., Sha, J., Foltz, S. M., Erova, T. E., Walberg, K. G., Baze, W. B., Suarez, G., Peterson, J. W. \& Chopra, A. K. (2009). Characterization of the rat pneumonic plague model: infection kinetics following aerosolization of Yersinia pestis CO92. Microbes Infect 11, 205-214.

Barton, G. M. \& Medzhitov, R. (2003). Toll-like receptor signaling pathways. Science 300, 1524-1525.

Berlato, C., Cassatella, M. A., Kinjyo, I., Gatto, L., Yoshimura, A. \& Bazzoni, F. (2002). Involvement of suppressor of cytokine signaling-3 as a mediator of the inhibitory effects of IL-10 on lipopolysaccharideinduced macrophage activation. J Immunol 168, 6404-6411.

Bingle, L. E., Bailey, C. M. \& Pallen, M. J. (2008). Type VI secretion: a beginner's guide. Curr Opin Microbiol 11, 3-8.

Carvalho-Castro, G. A., Lopes, C. O., Leal, C. A., Cardoso, P. G., Leite, R. C. \& Figueiredo, H. C. (2010). Detection of type III secretion system genes in Aeromonas hydrophila and their relationship with virulence in Nile tilapia. Vet Microbiol 144, 371-376.

Cascales, E. (2008). The type VI secretion toolkit. EMBO Rep 9, 735741.

Chopra, A. K. \& Houston, C. W. (1999). Enterotoxins in Aeromonasassociated gastroenteritis. Microbes Infect 1, 1129-1137.

Chopra, A. K., Xu, X., Ribardo, D., Gonzalez, M., Kuhl, K., Peterson, J. W. \& Houston, C. W. (2000). The cytotoxic enterotoxin of Aeromonas hydrophila induces proinflammatory cytokine production and activates arachidonic acid metabolism in macrophages. Infect Immun 68, 2808-2818.

Coombes, B. K., Valdez, Y. \& Finlay, B. B. (2004). Evasive maneuvers by secreted bacterial proteins to avoid innate immune responses. Curr Biol 14, R856-R867.

Das, S. \& Chaudhuri, K. (2003). Identification of a unique IAHP (IcmF associated homologous proteins) cluster in Vibrio cholerae and other proteobacteria through in silico analysis. In Silico Biol 3, 287300 .

Diacovich, L. \& Gorvel, J. P. (2010). Bacterial manipulation of innate immunity to promote infection. Nat Rev Microbiol 8, 117-128.

Diehl, S. \& Rincon, M. (2002). The two faces of IL-6 on Th1/Th2 differentiation. Mol Immunol 39, 531-536.
Dong, Q., Fan, R., Zhao, S. \& Wang, Y. (2009). Over-expression of SOCS-3 gene promotes IL-10 production by JEG-3 trophoblast cells. Placenta 30, 11-14.

Dramsi, S. \& Cossart, P. (2002). Listeriolysin O: a genuine cytolysin optimized for an intracellular parasite. J Cell Biol 156, 943-946.

Ernst, R. K., Yi, E. C., Guo, L., Lim, K. B., Burns, J. L., Hackett, M. \& Miller, S. I. (1999). Specific lipopolysaccharide found in cystic fibrosis airway Pseudomonas aeruginosa. Science 286, 1561-1565.

Filloux, A., Hachani, A. \& Bleves, S. (2008). The bacterial type VI secretion machine: yet another player for protein transport across membranes. Microbiology 154, 1570-1583.

Gordon, S. (2003). Alternative activation of macrophages. Nat Rev Immunol 3, 23-35.

Gordon, S. \& Taylor, P. R. (2005). Monocyte and macrophage heterogeneity. Nat Rev Immunol 5, 953-964.

Hajjar, A. M., Ernst, R. K., Tsai, J. H., Wilson, C. B. \& Miller, S. I. (2002). Human Toll-like receptor 4 recognizes host-specific LPS modifications. Nat Immunol 3, 354-359.

Henneke, P. \& Golenbock, D. T. (2004). Phagocytosis, innate immunity, and host-pathogen specificity. J Exp Med 199, 1-4.

Hornef, M. W., Wick, M. J., Rhen, M. \& Normark, S. (2002). Bacterial strategies for overcoming host innate and adaptive immune responses. Nat Immunol 3, 1033-1040.

Hume, D. A. (2006). The mononuclear phagocyte system. Curr Opin Immunol 18, 49-53.

Hume, D. A. (2008). Macrophages as APC and the dendritic cell myth. J Immunol 181, 5829-5835.

Janda, J. M. \& Abbott, S. L. (1998). Evolving concepts regarding the genus Aeromonas: an expanding Panorama of species, disease presentations, and unanswered questions. Clin Infect Dis 27, 332-344.

Janeway, C. A., Jr \& Medzhitov, R. (2002). Innate immune recognition. Annu Rev Immunol 20, 197-216.

Kawasaki, K., Ernst, R. K. \& Miller, S. I. (2004). 3-O-deacylation of lipid A by PagL, a PhoP/PhoQ-regulated deacylase of Salmonella typhimurium, modulates signaling through Toll-like receptor 4. J Biol Chem 279, 20044-20048.

Lejeune, D., Demoulin, J. B. \& Renauld, J. C. (2001). Interleukin 9 induces expression of three cytokine signal inhibitors: cytokineinducible SH2-containing protein, suppressor of cytokine signalling (SOCS)-2 and SOCS-3, but only SOCS-3 overexpression suppresses interleukin 9 signalling. Biochem J 353, 109-116.

Ma, A. T. \& Mekalanos, J. J. (2010). In vivo actin cross-linking induced by Vibrio cholerae type VI secretion system is associated with intestinal inflammation. Proc Natl Acad Sci U S A 107, 4365-4370.

Ma, A. T., McAuley, S., Pukatzki, S. \& Mekalanos, J. J. (2009). Translocation of a Vibrio cholerae type VI secretion effector requires bacterial endocytosis by host cells. Cell Host Microbe 5, 234-243.

Mantovani, A., Sica, A., Sozzani, S., Allavena, P., Vecchi, A. \& Locati, M. (2004). The chemokine system in diverse forms of macrophage activation and polarization. Trends Immunol 25, 677686.

McGuirk, P., McCann, C. \& Mills, K. H. (2002). Pathogen-specific T regulatory 1 cells induced in the respiratory tract by a bacterial molecule that stimulates interleukin 10 production by dendritic cells: a novel strategy for evasion of protective $\mathrm{T}$ helper type 1 responses by Bordetella pertussis. J Exp Med 195, 221-231.

Medzhitov, R. \& Janeway, C. A., Jr (1999). Innate immune induction of the adaptive immune response. Cold Spring Harb Symp Quant Biol 64, 429-435.

Moore, K. W., O'Garra, A., de Waal Malefyt, R., Vieira, P. \& Mosmann, T. R. (1993). Interleukin-10. Annu Rev Immunol 11, 165-190. 
Mosser, D. M. (2003). The many faces of macrophage activation. J Leukoc Biol 73, 209-212.

Mosser, D. M. \& Edwards, J. P. (2008). Exploring the full spectrum of macrophage activation. Nat Rev Immunol 8, 958-969.

Park, J. M., Greten, F. R., Li, Z. W. \& Karin, M. (2002). Macrophage apoptosis by anthrax lethal factor through p38 MAP kinase inhibition. Science 297, 2048-2051.

Plüddemann, A., Mukhopadhyay, S. \& Gordon, S. (2006). The interaction of macrophage receptors with bacterial ligands. Expert Rev Mol Med 8, 1-25.

Pujol, C. \& Bliska, J. B. (2005). Turning Yersinia pathogenesis outside in: subversion of macrophage function by intracellular Yersiniae. Clin Immunol 114, 216-226.

Pukatzki, S., McAuley, S. B. \& Miyata, S. T. (2009). The type VI secretion system: translocation of effectors and effector-domains. Curr Opin Microbiol 12, 11-17.

Ruckdeschel, K., Mannel, O. \& Schrottner, P. (2002). Divergence of apoptosis-inducing and preventing signals in bacteria-faced macrophages through myeloid differentiation factor 88 and IL-1 receptorassociated kinase members. J Immunol 168, 4601-4611.

Seifert, H. S. (1996). Questions about gonococcal pilus phase- and antigenic variation. Mol Microbiol 21, 433-440.

Sha, J., Pillai, L., Fadl, A. A., Galindo, C. L., Erova, T. E. \& Chopra, A. K. (2005). The type III secretion system and cytotoxic enterotoxin alter the virulence of Aeromonas hydrophila. Infect Immun 73, 6446-6457.

Sha, J., Wang, S. F., Suarez, G., Sierra, J. C., Fadl, A. A., Erova, T. E., Foltz, S. M., Khajanchi, B. K., Silver, A. \& other authors (2007). Further characterization of a type III secretion system (T3SS) and of a new effector protein from a clinical isolate of Aeromonas hydrophilaPart I. Microb Pathog 43, 127-146.

Sierra, J. C., Suarez, G., Sha, J., Foltz, S. M., Popov, V. L., Galindo, C. L., Garner, H. R. \& Chopra, A. K. (2007). Biological characterization of a new type III secretion system effector from a clinical isolate of Aeromonas hydrophila-Part II. Microb Pathog 43, 147-160.

Sierra, J. C., Suarez, G., Sha, J., Baze, W. B., Foltz, S. M. \& Chopra, A. K. (2010). Unraveling the mechanism of action of a new type III secretion system effector AexU from Aeromonas hydrophila. Microb Pathog 49, 122-134.

Sing, A., Roggenkamp, A., Geiger, A. M. \& Heesemann, J. (2002). Yersinia enterocolitica evasion of the host innate immune response by $\mathrm{V}$ antigen-induced IL-10 production of macrophages is abrogated in IL-10-deficient mice. J Immunol 168, 1315-1321.

Sturgill-Koszycki, S., Schlesinger, P. H., Chakraborty, P., Haddix, P. L., Collins, H. L., Fok, A. K., Allen, R. D., Gluck, S. L. \& Heuser, J.
(1994). Lack of acidification in Mycobacterium phagosomes produced by exclusion of the vesicular proton-ATPase. Science 263, 678-681.

Suarez, G., Sierra, J. C., Sha, J., Wang, S., Erova, T. E., Fadl, A. A., Foltz, S. M., Horneman, A. J. \& Chopra, A. K. (2008). Molecular characterization of a functional type VI secretion system from a clinical isolate of Aeromonas hydrophila. Microb Pathog 44, 344-361.

Suarez, G., Sierra, J. C., Erova, T. E., Sha, J., Horneman, A. J. \& Chopra, A. K. (2010). A type VI secretion system effector protein, VgrG1, from Aeromonas hydrophila that induces host cell toxicity by ADP ribosylation of actin. J Bacteriol 192, 155-168.

Sweet, C. R., Conlon, J., Golenbock, D. T., Goguen, J. \& Silverman, N. (2007). YopJ targets TRAF proteins to inhibit TLR-mediated NF- $\kappa$ B, MAPK and IRF3 signal transduction. Cell Microbiol 9, 2700-2715.

Tan, Y. W., Yu, H. B., Leung, K. Y., Sivaraman, J. \& Mok, Y. K. (2008). Structure of AscE and induced burial regions in AscE and AscG upon formation of the chaperone needle-subunit complex of type III secretion system in Aeromonas hydrophila. Protein Sci 17, 1748-1760.

Taylor, P. R., Martinez-Pomares, L., Stacey, M., Lin, H. H., Brown, G. D. \& Gordon, S. (2005). Macrophage receptors and immune recognition. Annu Rev Immunol 23, 901-944.

Thiefes, A., Wolf, A., Doerrie, A., Grassl, G. A., Matsumoto, K., Autenrieth, I., Bohn, E., Sakurai, H., Niedenthal, R. \& other authors (2006). The Yersinia enterocolitica effector YopP inhibits host cell signalling by inactivating the protein kinase TAK1 in the IL-1 signalling pathway. EMBO Rep 7, 838-844.

Uchiya, K., Barbieri, M. A., Funato, K., Shah, A. H., Stahl, P. D. \& Groisman, E. A. (1999). A Salmonella virulence protein that inhibits cellular trafficking. EMBO J 18, 3924-3933.

Viboud, G. I. \& Bliska, J. B. (2005). Yersinia outer proteins: role in modulation of host cell signaling responses and pathogenesis. Annu Rev Microbiol 59, 69-89.

Vilches, S., Jimenez, N., Tomas, J. M. \& Merino, S. (2009). Aeromonas hydrophila AH-3 type III secretion system expression and regulatory network. Appl Environ Microbiol 75, 6382-6392.

Xu, X. J., Ferguson, M. R., Popov, V. L., Houston, C. W., Peterson, J. W. \& Chopra, A. K. (1998). Role of a cytotoxic enterotoxin in Aeromonasmediated infections: development of transposon and isogenic mutants. Infect Immun 66, 3501-3509.

Zuany-Amorim, C., Sawicka, E., Manlius, C., Le Moine, A., Brunet, L. R., Kemeny, D. M., Bowen, G., Rook, G. \& Walker, C. (2002). Suppression of airway eosinophilia by killed Mycobacterium vaccaeinduced allergen-specific regulatory T-cells. Nat Med 8, 625-629.

Edited by: H. Hilbi 